\title{
Research on the Construction of Knowledge base for Existing residence Diagnosis and Repair
}

\author{
Xiao Wang ${ }^{1, a,{ }^{*}}$, Bing $\mathrm{Li}^{2, \mathrm{~b}}$, Yanwei $\mathrm{Li}^{1, \mathrm{c}}$ \\ ${ }^{1}$ School of Civil Engineering and Architecture, University of Jinan, Jinan 250022, China \\ ${ }^{2}$ Tongyuan Design Group Co., Ltd. Jinan 250101, China \\ axiaowang2010@126.com, blibing19832008@163.com, ccea_liyw@ujn.edu.cn
}

Keywords: Existing residence, Maintenance and update, Knowledge base.

\begin{abstract}
The knowledge base of diagnosis and repair of existing residence provides support for its maintenance and update. The paper aims at investigating the maintenance of existing residence as well as the decision-making and credibility of the renewal projects. Based on the knowledge base of diagnosis and repair of existing residence, the paper attempts to propose a fundamental structure for the diagnosis and repair of existing residence under the guidance of the knowledge base to construct an integrated framework to improve the efficiency of housing maintenance and update.
\end{abstract}

\section{Introduction}

China's residential construction has gradually changed from expansion in scale to a stage of quality improvement. The maintenance and update of existing residential buildings will become the "new norm" on the road of residential construction in China. However, the maintenance and renewal of the existing housing in China has just started, with the residential testing and maintenance industry in China still in the primary stage and the technical personnel not qualified enough. The diagnosis and decisions concerning deterioration of existing residence is usually based on individual experiences and expertise, coupled with the lack of information collection and classification of existing residence deterioration, makes it difficult to cope with the complexity, uncertainty and wide range of disciplines it involves. In addition, the corresponding maintenance supervision, management mechanism and backward means have resulted in low maintenance efficiency and quality.

In addition, compared with other industries, the traditional industry of residence maintenance and renewal lacks solid high-tech supports. There are relatively mature experiences and theories for the problems encountered in the detection, diagnosis, maintenance and repair engineering, but its complexity in procedures makes it not that accessible. At the same time, the current computer technology has been highly developed, with wide applications and researches in other fields, including the quality control platform for construction engineering supported by the knowledge base $^{[1]}$, health information management system for intelligent building ${ }^{[2]}$, digital platform for urban community greening transformation ${ }^{[3]}$. Collection and summary of current experiences of maintenance and updates in the computer processing would make it easier to access and share information, which could be applied to both the residence maintenance and updates to promote the development of both the residential maintenance industry and urban renewal and reconstruction theoretically and practically.

Therefore, this paper puts forward that by collecting and sorting out the information related to the deterioration of the existing residence, the diagnosis system integrating a number of experts could be introduced to construct the knowledge base for diagnosis and repair of existing residence based on information technologies and the "deterioration-cause-repair" framework. 


\section{Perception and Target of the Knowledge Base of Existing Residence Diagnosis and Repair}

\subsection{Perception of the knowledge base of existing residence diagnosis and repair}

A rational repair decision of the existing house should be based on the diagnosis and evaluation of the house's deterioration conditions. It can also be said that the collection, diagnosis and research of residential deterioration could aid in decisions on repair of the existing residential houses, which needs to be practical with assistance from specific technical methods and platform tools for strategy construction. Based on this, the existing residential diagnosis and repair system could be transplanted to the computer in the form knowledge base-a special computer program.

In this paper, the existing residence diagnosis and repair knowledge base refers to the collection and recording of information and data on the existing residence deterioration as well as the analysis of the deterioration. The construction of knowledge base, on the one hand, can integrate database on existing residence deterioration as well as diagnosis to form an information database; On the other hand, as an auxiliary tool for the diagnosis and evaluation of the existing residence, the scientific tools would enable the restoration decisions to be more rationale.

\subsection{Design Target of Knowledge Base}

\subsubsection{Information sharing}

The main and direct goal of the auxiliary knowledge base is to provide information for the maintenance and update of the existing residence. Therefore, the main aim is to establish an information database of deterioration of the existing residence. The storage and classification of information on the existing residence deterioration and repair will make the extraction and application of information more convenient and intuitive, serving as references for the future diagnosis and evaluation by relevant personnel and repair designers before final implementation of renewal projects. In the end, the whole system is expected to serve as a platform for diagnosis and information collection of existing residence degradation and repair, providing a fusion interface for multiple disciplines to exchange information.

\subsubsection{Improve repair efficiency and quality}

The knowledge base is serving as a tool to facilitate the maintenance and update of residential buildings. The goal is to assist related personnel in the diagnosis and analysis of the deterioration of residential buildings through the knowledge base in the preliminary planning stage of the maintenance and update of existing residential buildings so that the optimal approach for residence repair could be decided. The knowledge base should allow easy access to simplify the implementation procedures of renewal projects and improve its efficiency.

\subsubsection{System integration}

Participants in the knowledge base of residential diagnosis and repair need to fulfill functions including evaluation, repair design and maintenance. The system not only provide images and data on degradation diagnosis and assessment for related personnel to determine the cause, but also provide support for personnel to determine the optimal approach of repair, serving as references for maintenance technicians in the real scenario. In addition, with the improvement of the system and the accumulation of data, the knowledge base can also be used as the data basis for the government and industries involved to formulate the housing maintenance policy. The establishment of knowledge base system can meet the actual needs of all parties and allow integration of all departments concerned.

\section{System Design of the Knowledge Base of Diagnosis and Repair of Existing Residential Buildings}

\subsection{Basic structure of information processing}

The knowledge base platform is an open and extensible information system integrating data collection, management, analysis and expression, accessible to different subjects in different environments. It usually includes hardware, software, background data and users. Computer hardware includes all kinds of computer processing and terminal equipment, mainly responsible for processing and massive 
data storage in a short time and providing assistance. Software is responsible for receiving valid data and processing data correctly. It is a computer program system that supports the collection, storage, sorting, feedback and response to questions from users. Background data is the target of analysis and processing, the foundation of the knowledge base. The user is the target of service, who is allowed to retrieve and edit information.

The SQLSERVER database management system is selected as the platform of the knowledge base in this study, whose back-end database consists of the "basic information database of deterioration analysis", integrating diagnosis and repair information of existing houses on the Web browser as the unified display platform. The basic structure of knowledge base consists of display layer, logic layer and data layer. The display layer is mainly an interface for users to access the system for functional operation. It is mainly displayed through the Web browser for information query, input and display. The logic layer, or the second layer, lies between the display layer and the data layer, mainly responsible for reasoning and processing data requests from the display layer. While obtaining relevant data from the data layer, it also sends relevant instructions to the data layer. The data layer, or the third layer, is used for data management and implementation of instructions from the logic layer, mainly responsible for the storage, update, maintenance and management of data (Fig. 1).

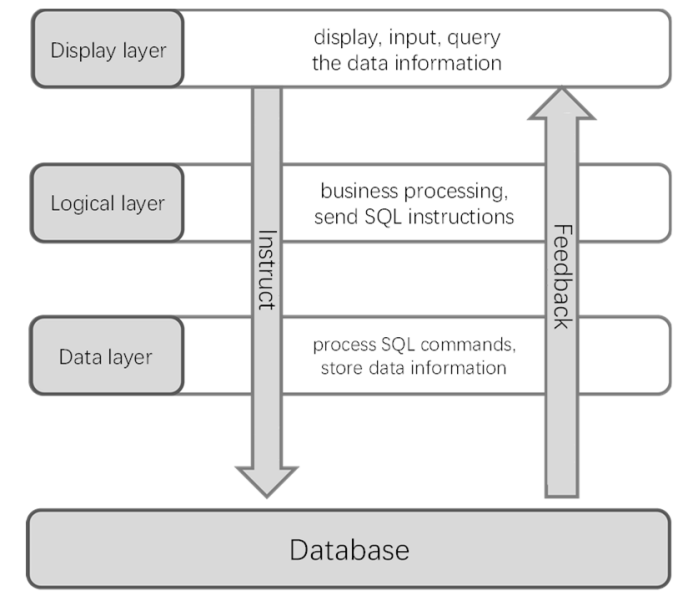

Fig. 1. Basic Structure of Information Processing.

\subsection{Data information}

Data information is the core in the construction of the knowledge base and the premise and foundation of the overall structure. In terms of information, it mainly includes two types of information, namely residence degradation and related images. The database establishment involves classification, sorting and input of the above information. The data information of residential deterioration mainly includes the structural form, construction age, number of floors of the residential unit, as well as deterioration images and the location of the unit. Residential deterioration information is mainly used to encode the deterioration images, whose coding number corresponds to the data information. Different codes imply corresponding data information. The coding code is made up of five numbers, representing "degradation + position + floor + structure form + construction year" to form the final database of deterioration information. The establishment of information database facilitates the edition of deterioration information, management and storage, as well as information retrieval through the SQL database management system.

\subsection{Design of the Knowledge Base}

Due to the complexity of renovation of existing residential houses, it is difficult for individuals to gain a thorough understanding of the field and make timely and effective treatment. At the same time, people also come to realize through practice that the significance of expertise in the diagnosis of housing deterioration, which is necessary for the effective repair of housing. Therefore, in the establishment of knowledge base, the "deterioration-diagnosis-repair" system is used as the basis of the knowledge base platform. 
The "deterioration-diagnosis-repair" system mainly includes three steps: collecting and sorting out deterioration (basic information database of deterioration); diagnosis and evaluation of deterioration (expert consultation and evaluation); decision-making for optimal repair approach.

1)Collection and sorting deterioration. The first step is to collect, classify and store the basic information of deterioration, which is matched and combined with images to form a "pathological property list" for output. "Pathological property list" is the basis for the diagnosis of existing residence deterioration, and the basic template for the information collation in the design of knowledge base system. The table includes the basic properties and visual images of a single unit of deterioration, including the construction age, the structural form, coding of deterioration, the location, the description of deterioration, and deterioration images.

2)Diagnosis and assessment of deterioration. As mentioned above, since the diagnosis of deterioration in existing houses involves multiple fields and disciplines, the effective restoration of houses must rely on rich engineering experience. Therefore, the second step consists of expert consultation and evaluation of the causes of deterioration.

3)Decision-making on the optimal repair method. The third step is to integrate multidisciplinary knowledge and information through expert consultation to develop alternative repair methods and then determine the optimal repair plan based on scientific analysis. The above information is collected in the database for storage, which is conducive to scientific analysis and decision-making of the repair plan and the ultimate establishment of the "deterioration-diagnosis-repair" system.

\section{Conclusion}

It is difficult for a single discipline or subject to study the maintenance and renewal of existing houses, which is complex and time-consuming. The maintenance and renewal of the existing residential buildings depends on interdisciplinary cooperation and participation of personnel from multiple fields. This paper combines the knowledge base with the maintenance and update of the existing residence, and explores the construction of the knowledge base for the diagnosis and repair of existing residence in order to make science-based decisions and meet the growing needs of housing maintenance.

\section{Acknowledgements}

This research was financially supported by Shandong Provincial Natural Science Foundation (ZR2017BEE074).

\section{References}

[1] Zhang, J. Study of construction engineering quality control platform based on repository. M.E, Huazhong University of Science and Technology, Wuhan, China, 2005.

[2] He, Y.\& Han, C. Discussion on Construction of Intelligent Building Health Information Service Management System Based on IOT and Big Data, Construction Economy2015; 5:101-106.

[3] Editorial Committee of Retrofitting of Existing Buildings Yearbook. (2013) Retrofitting of Existing Buildings Yearbook. China Architecture\& Building Press, Beijing. 